\title{
Clinical Relapse of Anti-AMPAR Encephalitis Associated with Recurrence of Thymoma
}

\author{
Tsubasa Omi ${ }^{1}$, Makoto Kinoshita ${ }^{2}$, Akira Nishikawa $^{2}$, Takahito Tomioka ${ }^{1}$, \\ Kenichi Ohmori ${ }^{3}$, Kei Fukada ${ }^{2}$ and Hidenori Matsunaga ${ }^{1}$
}

\begin{abstract}
:
We report a rare case of anti- $\alpha$-amino-3-hydroxy-5-methyl-4-isoxazolepropionic acid receptor (AMPAR) encephalitis presenting clinical relapse in association with recurrence of thymoma. Anti-AMPAR encephalitis is an autoimmune-mediated neurological disease, frequently accompanied by the presence of neoplasms, thus comprising the spectrum of paraneoplastic syndrome. A patient had been in remission for 34 months showed clinical relapse 3 months after the detection of recurrent thymoma. Clinical relapse of anti-AMPAR encephalitis after the recurrence of an initially detected neoplasm has not been previously reported. Our case therefore highlights the pathogenic relevance of specific tumor antigens as a trigger of anti-AMPAR antibody production and induction of the disease.
\end{abstract}

Key words: anti-AMPAR encephalitis, thymoma, paraneoplastic syndrome

(Intern Med 57: 1011-1013, 2018)

(DOI: 10.2169/internalmedicine.9682-17)

\section{Introduction}

Patients with anti- $\alpha$-amino-3-hydroxy-5-methyl-4isoxazolepropionic acid receptor (AMPAR) encephalitis commonly present with limbic dysfunction (1-3). Approximately $60 \%$ of patients possess pathologically or radiologically proven tumors, indicating the pathogenic relevance of the presence of tumor antigens and autoantibody production (4). Although patients with anti-AMPAR encephalitis reportedly have a high rate of neurological relapse $(4,5)$, the precise mechanisms underlying clinical relapse induction remain unknown.

We herein report the case of a patient with anti-AMPAR encephalitis who presented with clinical relapse associated with thymoma recurrence. Our case supports the hypothesis that specific tumor antigens may trigger anti-AMPAR antibody production, thus contributing to the development of the disease.

\section{Case Report}

A 34-year-old woman presented with acute onset of confusion, agitation, and gait disturbance. On the initial neurological examination, she was disorientated, showed stereotyped behaviors, followed by partial seizure of the right arm. Routine blood and cerebrospinal fluid (CSF) analyses were normal. Brain magnetic resonance imaging (MRI) identified high-intensity signals on fluid-attenuated inversion recovery images in the lower region of the left caudate nucleus (Figure A). Whole-body computed tomography (CT) revealed a 5.4-cm anterior mediastinal mass. An electroencephalogram analysis indicated focal epileptic discharges at the left temporal-parietal region. She was provisionally diagnosed with autoimmune encephalitis associated with thymoma. Corticosteroid therapy (methylprednisolone, $1 \mathrm{~g}$ /day for 3 days) was initiated on day 1 of hospitalization, and thymectomy was performed on the following day. A pathological analysis revealed that the resected thymoma was consistent with the type B3 phenotype.

Psychomotor excitation appeared on postoperative day 18;

\footnotetext{
${ }^{1}$ Department of Psychiatry, Osaka General Medical Center, Japan, ${ }^{2}$ Department of Neurology, Osaka General Medical Center, Japan and ${ }^{3}$ Department of Thoracic Surgery, Rinku General Medical Center, Japan

Received: June 19, 2017; Accepted: July 25, 2017; Advance Publication by J-STAGE: December 8, 2017
}

Correspondence to Dr. Tsubasa Omi, omi@gh.opho.jp 


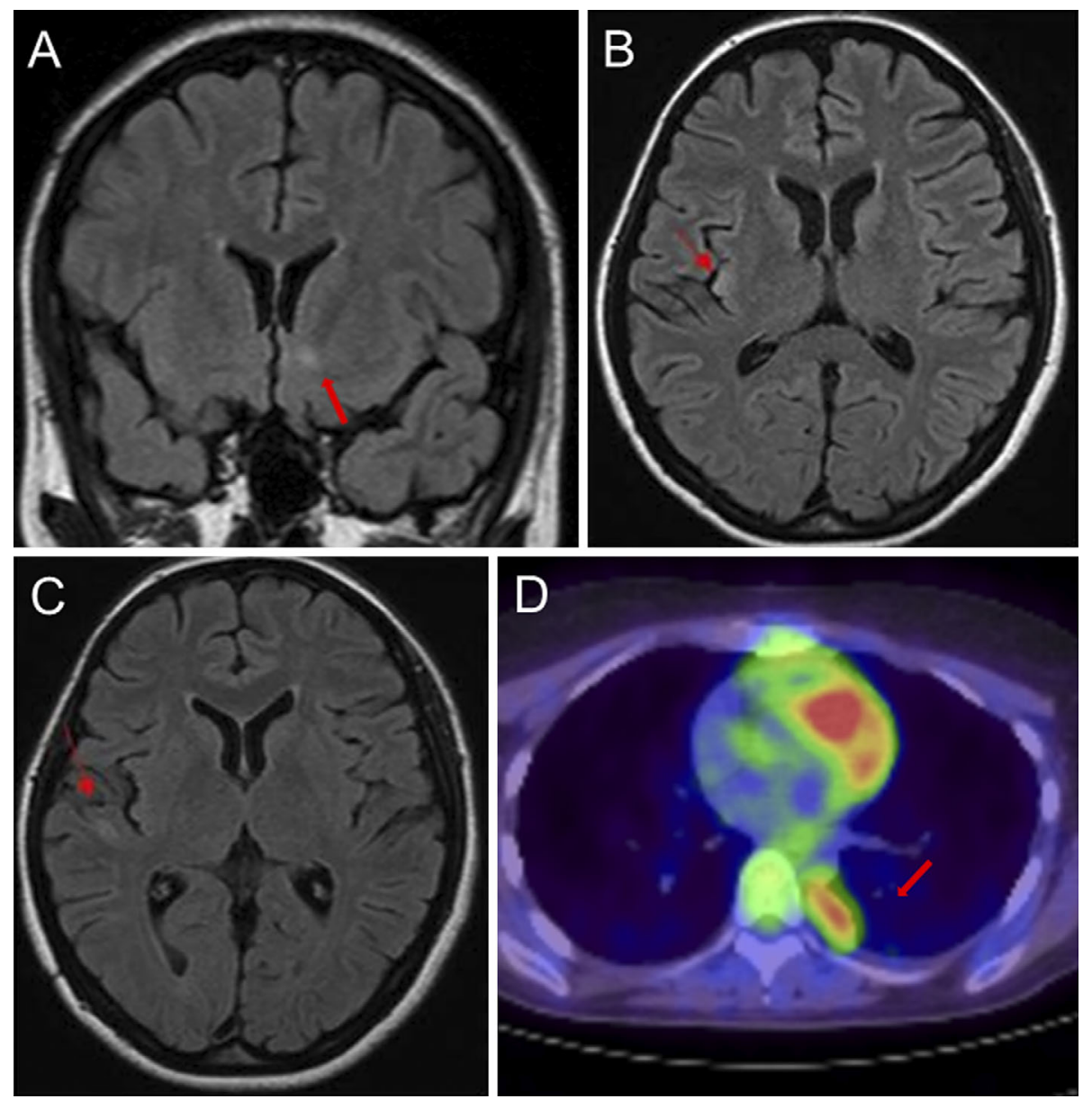

Figure. Brain magnetic resonance imaging and positron emission tomography/computed tomography (PET/CT) of the thorax. (A) On admission, a hyperintense lesion was identified in the lower region of the left caudate nucleus by fluid-attenuated inversion recovery (FLAIR) imaging. (B) On day 14 of hospitalization, a new lesion was identified in the right insula by FLAIR imaging. (C) On day 54 of hospitalization, a new lesion was identified in the right temporal lobe by FLAIR imaging. (D) Preoperative chest PET/CT images showed a 1.2-cm mass at the base of the right lung, which indicated thymoma recurrence.

thus, psychotropic drug treatments were initiated. Samples of her serum and CSF were positive for anti-AMPAR antibodies but negative for anti-N-methyl-D-aspartate receptor, contactin-associated protein 2 , and leucine-rich glioma inactivated 1 antibodies.

The initial MRI lesions resolved after thymectomy and corticosteroid therapy, but brain MRI performed on day 14 and 54 of hospitalization demonstrated new lesion formation in the right insula and right temporal lobe, respectively (Figure B and C). Pulse corticosteroid therapy was repeated five times until day 98 of hospitalization, and high-dose oral corticosteroid therapy was initiated on day 100. Even after the MRI lesions recovered and the psychomotor excitation improved, she exhibited depressive symptoms for approximately six months. The patient was discharged on day 310 of hospitalization.

As an outpatient, she received $10 \mathrm{mg}$ of prednisone per day for 10 months, which was subsequently discontinued. Her depressive symptoms continued during the clinical follow-up. Thirty-four months after hospital discharge, whole-body positron emission tomography computed tomography (PET/CT) identified a 1.2-cm mass at the base of the right lung, indicating thymoma recurrence (Figure D). Three months after resection of the pleural dissemination, acute memory deficits appeared. She was not able to recall her daily activities after a couple of hours and showed mild executive dysfunction. Her Mini-Mental State Examination (MMSE) score on admission was 24. After diagnosing her with a clinical relapse of anti-AMPAR encephalitis, she underwent corticosteroid pulse therapy twice, and oral corticosteroid therapy (20 mg per day) was initiated. Thirty-two days after starting steroid therapy, her memory deficits showed gradual improvement, and her MMSE score recovered to 29. She was discharged on day 43 after hospitalization.

\section{Discussion}

Several studies have reported high rates of neurological relapse in patients with anti-AMPAR encephalitis $(4,5)$. 
Höftberger et al. reported that none of the patients receiving aggressive therapy such as chemotherapy or rituximab showed relapse, while 6 out of 18 patients who did not go through these treatment regimens developed further relapses, attributing the discrepancy to the therapeutic strategies applied (4).

It is noteworthy that all of the reported cases of relapse in the literature occurred without tumor recurrence $(4,5)$. Therefore, a case of anti-AMPAR encephalitis with concomitant oncogenic relapse has not been previously described. Our case is unique in that there was a chronological relationship between the clinical relapse and thymoma recurrence.

The presence of neuronal antigens in neoplasm and susceptibility to autoimmune disorders are postulated as potential triggers for anti-neuronal antibody production (5). Lai et al. demonstrated the expression of GluR1 and GluR2 subunits in tumors of patients with anti-AMPAR encephalitis (5). In addition, the majority of patients have been shown to possess either other autoimmune diseases or certain autoantibodies (5).

Although our patient did not turn out to be positive for other autoantibodies and showed no concomitant autoimmune diseases, the presence of recurrent thymoma before the clinical relapse strongly supports the hypothesis that neuronal antigens expressed in tumor cells served as a pivotal trigger to elicit anti-AMPAR antibodies and further promoted the development of the disease. We have not evaluated the expression of AMPAR in the resected tumor. Therefore, it remains to be determined in a future study whether the recurrence of encephalitis in our patient was due to the aberrant expression of AMPAR within the recurrent thymoma, or if instead the recurrent thymoma itself elicited autoimmune responses, leading to the excess production of pathogenic anti-AMPAR antibodies.

Our case highlight the pathogenic link between the presence of tumor antigens and anti-AMPAR antibody production $(5,6)$ and indicates the need to look for occult neoplasms in cases of clinical relapses of anti-AMPAR en- cephalitis.

The authors state that they have no Conflict of Interest (COI).

\section{Acknowledgement}

We thank Dr. Josep Dalmau (ICREA-IDIBAPS, Service of Neurology, Hospital Clínic, University of Barcelona, Barcelona, Spain, and University of Pennsylvania, Philadelphia), Dr. Takashi Kanbayashi (Department of Neuropsychiatry, Akita University Graduate School of Medicine, Hondo, Akita, Japan), and Dr. Kou Tsutsui (Department of Neuropsychiatry, Akita University Graduate School of Medicine, Hondo, Akita, Japan) for performing the autoimmune-serologic tests.

\section{Financial Support}

The Osaka Medical Research Foundation for Intractable Diseases

\section{References}

1. Bataller L, Galiano R, García-Escrig M, et al. Reversible paraneoplastic limbic encephalitis associated with antibodies to the AMPA receptor. Neurology 74: 265-267, 2010.

2. Joubert B, Kerschen P, Zekeridou A, et al. Clinical spectrum of encephalitis associated with antibodies against the $\alpha$-amino- 3 hydroxy-5-methyl-4-isoxazolepropionic acid receptor: case series and review of the literature. JAMA Neurol 72: 1163-1169, 2015.

3. Graus F, Boronat A, Xifró X, et al. The expanding clinical profile of anti-AMPA receptor encephalitis. Neurology 74: 857-859, 2010.

4. Höftberger R, van Sonderen A, Leypoldt F, et al. Encephalitis and AMPA receptor antibodies: novel findings in a case series of 22 patients. Neurology 84: 2403-2412, 2015.

5. Lai M, Hughes EG, Peng X, et al. AMPA receptor antibodies in limbic encephalitis alter synaptic receptor location. Ann Neurol 65: 424-434, 2009.

6. Zekeridou A, McKeon A, Lennon VA. Frequency of synaptic autoantibody accompaniments and neurological manifestations of thymoma. JAMA Neurol 73: 853-859, 2016.

The Internal Medicine is an Open Access article distributed under the Creative Commons Attribution-NonCommercial-NoDerivatives 4.0 International License. To view the details of this license, please visit (https://creativecommons.org/licenses/ by-nc-nd/4.0/).

\section{(C) 2018 The Japanese Society of Internal Medicine} Intern Med 57: 1011-1013, 2018 